\title{
Gene-expression changes in knee-joint tissues with aging and menopause: implications for the joint as an organ
}

\author{
Natalie C Rollick' \\ Devin B Lemmex' \\ Yohei Ono',2 \\ Carol R Reno' \\ David A Hart' \\ Ian KY Lo' \\ Gail M Thornton ${ }^{1,3}$
}

IMcCaig Institute for Bone and Joint

Health, Section of Orthopaedic

Surgery, Department of Surgery,

University of Calgary, Calgary, AB,

Canada; ${ }^{2}$ Department of Orthopaedic

Surgery, Nagoya University Graduate

School of Medicine, Nagoya, Japan;

${ }^{3}$ Department of Orthopaedics,

University of British Columbia,

Vancouver, BC, Canada
This article was published in the following Dove Press journal:

Clinical Interventions in Aging

\begin{abstract}
Background: When considering the "joint as an organ", the tissues in a joint act as complementary components of an organ, and the "set point" is the cellular activity for homeostasis of the joint tissues. Even in the absence of injury, joint tissues have adaptive responses to processes, like aging and menopause, which result in changes to the set point.

Purpose: The purpose of this study in a preclinical model was to investigate age-related and menopause-related changes in knee-joint tissues with the hypothesis that tissues will change in unique ways that reflect their differing contributions to maintaining joint function (as measured by joint laxity) and the differing processes of aging and menopause.
\end{abstract}

Methods: Rabbit knee-joint tissues from three groups were evaluated: young adult (gene expression, $n=8$; joint laxity, $n=7$; water content, $n=8$ ), aging adult (gene expression, $n=6$; joint laxity, $n=7$; water content, $n=5$ ), and menopausal adult (gene expression, $n=8$; joint laxity, $n=7$; water content, $\mathrm{n}=8$ ). Surgical menopause was induced with ovariohysterectomy surgery and gene expression was assessed using reverse-transcription quantitative polymerase chain reaction.

Results: Aging resulted in changes to 37 of the 150 gene-tissue combinations evaluated, and menopause resulted in changes to 39 of the 150 . Despite the similar number of changes, only eleven changes were the same in both aging and menopause. No differences in joint laxity were detected comparing young adult rabbits with aging adult rabbits or with menopausal adult rabbits.

Conclusion: Aging and menopause affected the gene-expression patterns of the tissues of the knee joint differently, suggesting unique changes to the set point of the knee. Interestingly, aging and menopause did not affect knee-joint laxity, suggesting that joint function was maintained, despite changes in gene expression. Taken together, these findings support the theory of the joint as an organ where the tissues of the joint adapt to maintain joint function.

Keywords: knee, aging, surgical menopause, joint as an organ

\section{Introduction}

In the early 2000s, the idea to consider the "joint as an organ" was formalized, with Lo et $\mathrm{al}^{1}$ using the concept to describe the function and homeostasis of diarthrodial joints and Frank et $\mathrm{al}^{2}$ further developing the concept to explain how joints adapt to influences, including injury, aging, and hormones, and then how treatments could target the changing "set points". Tissues in a joint act as complementary components of an organ, and the interactive set point is the cellular activity for homeostasis of the joint tissues under the current loading environment of the joint. ${ }^{2}$

The prevalence of knee-joint pain increases with aging and with menopause. ${ }^{3}$ The model of a physiologic organ to conceptualize the knee allows the consideration of each

\footnotetext{
Correspondence: Gail M Thornton McCaig Institute for Bone and Joint Health, Section of Orthopaedic Surgery, Department of Surgery, University of Calgary, 434 Heritage Medical Research Building, 3330 Hospital Drive NW, Calgary, AB T2N 4NI, Canada Tel +l 4032202296

Email gail.thornton@ucalgary.ca
} 
component of the joint as an important contributor to overall joint function. Furthermore, it is useful for contemplation of all components together as a single time snapshot of joint function; in other words, an equilibrium set point. ${ }^{2}$ Even in the absence of injury, the properties of joint tissues undergo adaptive changes secondary to age, genetics, sex (hormones), and environment (biological, mechanical). ${ }^{2}$ Investigation of how the set point changes under different conditions, eg, aging and menopause, is important to provide a comparative baseline for injury and degenerative profiles. While there are substantial biomechanical and molecular data on the knee, the majority of studies have focused on degenerative and injury states, such as osteoarthritis (OA) or following anterior cruciate ligament (ACL) injury. It is important to remember that neither aging nor menopause are synonymous with degeneration, and studies that investigate degenerative states must compare to an appropriate baseline. Careful consideration of aging and menopause is critical as our population ages, with women older than men, eg, 2016 estimates indicate that the median age in Japan is 48.3 years for females and 45.6 years for males, in Canada it is 43.3 years for females and 40.6 years for males, and in the US it is 39.3 years for females and 36.6 years for males. ${ }^{4}$ The current study uses a preclinical model to investigate how the set point changes with aging and with menopause by examining the resulting molecular changes to the knee-joint tissues that are the complementary components of the joint as an organ.

As age and female sex are risk factors for $\mathrm{OA},{ }^{5-7}$ it is not surprising that the joint-as-an-organ model has more recently been utilized by Loeser et $\mathrm{al}^{8}$ to synthesize the pathological changes in knee-joint tissues in the setting of OA. The use of this model allowed consideration of the individual markers and tissues, as well as the entire joint, indicating that changes in cartilage, ligaments, menisci, and synovium are important in the clinical syndrome of OA. Clinical evidence of the changed set point due to aging is supported by the observation that OA changes appear sooner in older patients with knee injury than in younger patients with the same knee injury. ${ }^{9}$

Estrogen receptors have been identified in human articular cartilage ${ }^{10}$ and estrogen and progesterone receptors have been identified in human synovium ${ }^{11,12}$ and human ligament, ${ }^{13,14}$ making these tissues susceptible to hormonal changes, including those in menopause. The knee-joint tissues of interest in this study were articular cartilage (AC; femoral condyle [FC AC] and tibial plateau [TP AC]), menisci (medial [MM] and lateral [LM]), synovium (Syn), intra-articular ligaments (ACL and posterior cruciate ligament [PCL]), extra-articular ligaments (medial collateral ligament [MCL] and lateral collateral ligament $[\mathrm{LCL}])$, and the patellar tendon (PT). The molecular markers with aging and menopause that we investigated included collagens (collagen I, collagen III, and collagen V), proteoglycans (biglycan, decorin, and lubricin), hyaluronan synthase 2 (HAS2), matrix metalloproteinases (MMP1, MMP3, $M M P 13)$, tissue inhibitors of metalloproteinases (TIMP1, TIMP2, TIMP3), and the hormone receptors - estrogen receptor $(E R)$ and progesterone receptor $(P R)$. The purpose of this study was to investigate age-related and menopause-related changes in knee-joint tissues with the hypothesis that tissues will change in unique ways that reflect their differing contributions to maintaining joint function (as measured by joint laxity) and the differing processes of aging and menopause.

\section{Methods}

In this study, approved by the University of Calgary Animal Care Committee, knee-joint tissues underwent molecular assessment from young adult ( 1 year old, $n=8$ ), aging adult ( 3 years old, $n=6$ ), and menopausal adult ( 1 year old, 14 weeks postovariohysterectomy, $n=8$ ) female New Zealand White rabbits, and all experiments were performed following University of Calgary Animal Care Committee and Canadian Council on Animal Care guidelines. Although our previous studies investigated the MCL only, ${ }^{15,16}$ in the current study various knee-joint tissues were investigated: AC (FC AC and TP AC), menisci (MM and LM), Syn, intra-articular ligaments (ACL and PCL), extra-articular ligaments (MCL and LCL), and PT. Surgical menopause was induced with ovariohysterectomy surgery removing the ovaries and uterus. ${ }^{16}$ Reverse-transcription quantitative polymerase chain reaction (RT-qPCR) was used to evaluate mRNA levels for collagens (collagen I, III, and V), proteoglycans (biglycan, decorin, and lubricin), HAS2, matrix metalloproteinases (MMP1, -3, and -13), tissue inhibitors of metalloproteinases (TIMP1, -2, and -3$)$, hormone receptors $(E R, P R)$, and the housekeeping gene $18 S$ (Table 1). Briefly, after tissues had been processed, total RNA was isolated and then quantified on a Turner 450 fluorescence spectrofluorometer (Barnstead Thermolyne, Dubuque, IA, USA) with a standard of calf liver ribosomal RNA. ${ }^{15,16}$ Total RNA ( $\left.1 \mu \mathrm{g}\right)$ was reverse-transcribed using an Omniscript RT kit (Qiagen Inc., Germantown, MD, USA), and then aliquots were used in real-time RT-qPCR using primers for the target genes (Table 1). Using an iQ SYBR Green Supermix (Bio-Rad, Hercules, CA, USA), RT-qPCR was carried out in an iCycler (Bio-Rad). Values for the target genes were normalized to corresponding $18 S$ values.

In addition, intact knee joints from young adult $(n=7)$, aging adult $(\mathrm{n}=7)$, and menopausal adult $(\mathrm{n}=7)$ rabbits underwent mechanical assessment in an MTS system (MTS Systems Corporation, Minneapolis, MN, USA) where the 
Table I Primer sequences for reverse-transcription quantitative polymerase chain reaction

\begin{tabular}{|c|c|c|c|c|}
\hline Gene & Forward & Reverse & Size & Source/GenBank accession number \\
\hline Collagen I & gatgcgttccagttcgagta & ggtcttccggtggtcttgta & 312 & $\begin{array}{l}\text { Email communication with WW Kao } \\
\text { (University of Cincinnati, Cincinnati, OH, USA) }\end{array}$ \\
\hline Collagen III & ttataaaccaacctcttcct & tattatagcaccattgagac & 255 & $\begin{array}{l}\text { Email communication with E Vuorio } \\
\text { (University of Turku, Turku, Finland) }\end{array}$ \\
\hline Collagen V & gaggagaaccaggaataacc & gcacctttctctccgatgcc & 215 & Consensus sequences from GenBank \\
\hline Biglycan & gatggcctgaagctcaa & ggttgttgaagaggctg & 406 & Consensus sequences from GenBank \\
\hline Decorin & tgtggacaatggttctctgg & ccacattgcagttaggttcc & 419 & GenBank S76584 \\
\hline Lubricin & gaacgtgctataggaccttc & cagactttggataaggtctgcc & 287 & GenBank NM_00 I I 27709 \\
\hline HAS2 & ggccggtcgtctcaaattca & ccaccccatttttgcatgat & 687 & GenBank NM_005328 \\
\hline$M M P I$ & gcagttgagaagctgaagca & ccatcaatgtcatcctgagc & 587 & GenBank AH005676 \\
\hline MMP3 & gccaagagatgctgttgatg & aggtctgtgaaggcgttgta & 363 & GenBank M25664 \\
\hline MMPI3 & ttcggcttagaggtgacagg & actcttgccggtgtaggtgt & 527 & GenBank AF05920I \\
\hline TIMPI & gcaactccgaccttgtcatc & agcgtaggtcttggtgaagc & 326 & GenBank J074 I I 2 \\
\hline TIMP2 & gtagtgatcagggccaaag & ttctctgtgacccagtccat & 416 & Consensus sequences from GenBank \\
\hline TIMP3 & tctgcaactccgacatcgtg & cggatgcaggcgtagtgtt & 454 & Consensus sequences from GenBank \\
\hline$E R$ & gtgtctgtgatcttgtcc & ctccatgatcaggtccac & 341 & Consensus sequences from GenBank \\
\hline$P R$ & ccacagtacagcttcgagtc & gaggacaccataatgacagc & 84 & GenBank NM_00 I082267 \\
\hline 185 & tggtcgctggctcctctcc & cgcctgctgccttccttgg & 360 & GenBank NR_003286.2 \\
\hline
\end{tabular}

Abbreviations: ER, estrogen receptor; HAS, hyaluronan synthase; MMP, matrix metalloproteinase; PR, progesterone receptor; TIMP, tissue inhibitor of metalloproteinase.

knee joint was mounted at $70^{\circ}$ of flexion and underwent two compression-tension cycles from $-5 \mathrm{~N}$ to $2 \mathrm{~N}$ at $1 \mathrm{~mm} / \mathrm{min}$. Joint laxity was defined as the displacement of the intact joint between $-5 \mathrm{~N}$ and $2 \mathrm{~N}{ }^{17}$

Water content was assessed for the ACL, PCL, MCL, LCL, and PT from young adult $(n=8)$, aging adult $(n=5)$, and menopausal adult $(n=8)$ rabbits. Tissue water content was expressed as a percentage: difference between the wet weight and the dry weight divided by the wet weight. ${ }^{15,16}$

Data were analyzed using a Student's $t$-test or a Mann-Whitney $U$ test to compare tissues from young adult rabbits to aging adult rabbits and young adult rabbits to menopausal adult rabbits.

\section{Results}

\section{Aging}

In comparisons of aging adult to young adult rabbits, mRNA levels for collagen $I$ were increased in FC AC, but collagen $V$ was decreased in TP AC $(P \leq 0.032$, Table 2 , Figures S1 and S2). In the LM, mRNA levels for collagen I were increased with aging, and in the MM, mRNA levels for collagen III and collagen $V$ were increased with aging

Table 2 Changes in mRNA levels with aging

\begin{tabular}{|c|c|c|c|c|c|c|c|c|c|c|}
\hline Gene & FC AC & TP AC & MM & LM & Syn & $A C L$ & PCL & MCL & LCL & PT \\
\hline Collagen I & $\uparrow$ & & & $\uparrow$ & & & & & & \\
\hline Collagen III & & & $\uparrow$ & & & & & & & \\
\hline Collagen V & & $\downarrow$ & $\uparrow$ & & & & & & & \\
\hline Biglycan & & $\downarrow$ & $\uparrow$ & & $\downarrow$ & & & & & \\
\hline Decorin & & & $\uparrow$ & & & & & & & \\
\hline Lubricin & & & & & & & & $\uparrow$ & & \\
\hline HAS2 & & $\downarrow$ & & $\downarrow$ & & & & & $\uparrow$ & \\
\hline$M M P I$ & $\uparrow$ & $\uparrow$ & & & & & & $\downarrow$ & & \\
\hline MMP3 & & & $\uparrow$ & & & & & & $\downarrow$ & \\
\hline$M M P / 3$ & & $\uparrow$ & & & & & & & & \\
\hline TIMPI & $\uparrow$ & $\uparrow$ & & & & $\uparrow$ & $\uparrow$ & & & \\
\hline TIMP2 & & & $\uparrow$ & & & & & & & $\downarrow$ \\
\hline TIMP3 & $\uparrow$ & & & & & $\uparrow$ & $\uparrow$ & $\uparrow$ & & \\
\hline$E R$ & $\uparrow$ & & & & & & & $\downarrow$ & & \\
\hline$P R$ & $\uparrow$ & $\uparrow$ & $\uparrow$ & & & $\uparrow$ & $\uparrow$ & & & $\uparrow$ \\
\hline
\end{tabular}

Notes: Increase $(\uparrow)$ and decrease $(\downarrow)$ comparing tissues from aging adult rabbits to young adult rabbits $(P \leq 0.059)$. Summarizes the data shown in Figures $S 1$ and $S 2$.

Abbreviations: $A C L$, anterior cruciate ligament; ER, estrogen receptor; FC AC, femoral condyle articular cartilage; HAS, hyaluronan synthase; $L C L$, lateral collateral ligament; LM, lateral meniscus; MMP, matrix metalloproteinase; MCL, medial collateral ligament; MM, medial meniscus; PCL, posterior cruciate ligament; PR, progesterone receptor; PT, patellar tendon; Syn, synovium; TP AC, tibial plateau articular cartilage; TIMP, tissue inhibitor of metalloproteinase. 

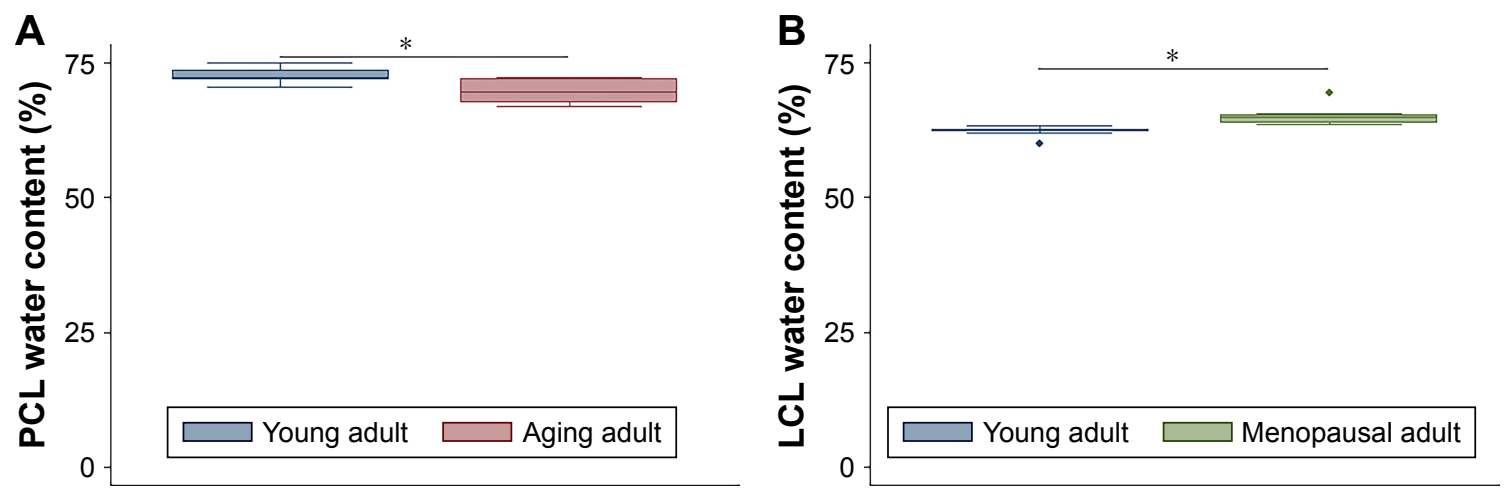

Figure I Water content: $(\mathbf{A})$ with aging in the posterior cruciate ligament (PCL); (B) with menopause in the lateral collateral ligament ( $\mathrm{LCL})$. Notes: $* P \leq 0.019$. The diamond symbol on the box plot indicates an outlier.

$(P \leq 0.008$, Table 2). Biglycan mRNA levels were decreased in the TP AC and Syn and increased in the MM, along with decorin ( $P \leq 0.03$, Table 2). Lubricin mRNA levels were increased in the MCL with aging $(P=0.028$; Table 2). mRNA levels for HAS2 were decreased in TP AC and LM, but increased in LCL with aging $(P \leq 0.016)$. mRNA levels for $M M P 1$ were increased in the $\mathrm{AC}$ (both $\mathrm{FC} \mathrm{AC}$ and TP AC; $P \leq 0.022)$ but decreased in the MCL $(P=0.059)$. mRNA levels for $M M P 13$ were increased in TP AC, and for $M M P 3$ were increased in MM but decreased in the LCL $(P \leq 0.032)$. mRNA levels for TIMPl were increased in FC AC, TP AC, $\mathrm{ACL}$, and PCL and for TIMP3 increased in the FC AC, ACL, PCL, and MCL with aging $(P \leq 0.059)$. mRNA levels for TIMP2 were increased in MM but decreased in PT with aging $(P \leq 0.032)$. mRNA levels for $E R$ were increased in FC AC but decreased in MCL with aging ( $P \leq 0.022$; Table 2$)$. mRNA levels for $P R$ were increased in the FC AC, MM, ACL, PCL, PT $(P \leq 0.028)$, and TP AC $(P=0.055)$ with aging. Only the PCL demonstrated decreased water content with aging $(P=0.019$, Figure $1 \mathrm{~A})$. No differences in joint laxity were detected between intact knee joints from young adult rabbits and aging adult rabbits (Figure 2A).

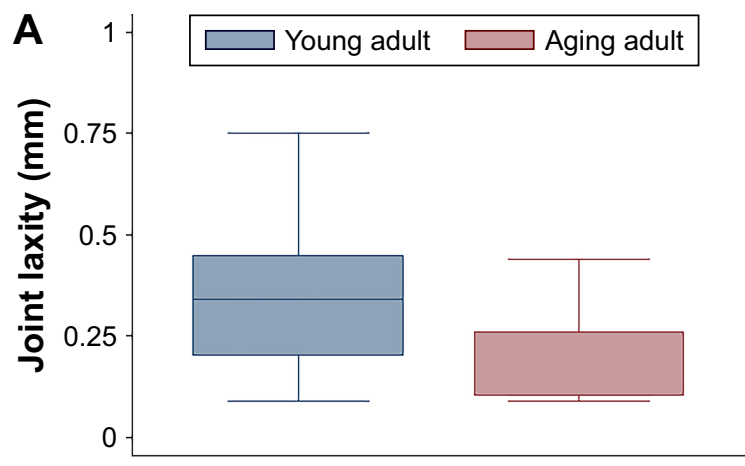

Figure 2 Joint laxity: (A) with aging; (B) with menopause.

\section{Menopause}

In comparisons of menopausal adult to young adult rabbits, mRNA levels for collagen $V$ were decreased for TP AC $(P=0.021$, Table 3, Figures S3 and S4). mRNA levels for collagen I were increased in MM, Syn, PCL, MCL, and PT, for collagen III were increased in MM, LM, PCL, and PT, and for collagen $V$ were increased in MM and PCL with menopause $(P \leq 0.046$, Table 3$)$. mRNA levels for decorin were increased in TP AC $(P=0.056)$, and for biglycan were increased in PT, but decreased in Syn $(P \leq 0.015$, Table 3$)$. mRNA levels for lubricin were increased in FC AC, Syn, ACL, PCL, and MCL, and for HAS2 were decreased in TP AC and Syn ( $P \leq 0.049$, Table 3). mRNA levels for $M M P 1$, $M M P 3$, and $M M P 13$ were increased in the PCL with menopause $(P \leq 0.007)$. mRNA levels for $M M P 1$ were increased in Syn and PT $(P \leq 0.059)$, but decreased in LCL with menopause $(P=0.047)$. mRNA levels for TIMP1 were increased in Syn and ACL $(P \leq 0.054)$, but decreased in LM $(P=0.012)$. mRNA levels for TIMP2 were decreased in LM, Syn, and $\mathrm{PT}$, and for TIMP 3 were increased in PCL with menopause $(P \leq 0.048)$. mRNA levels for $E R$ were decreased in LCL, but increased in PCL, and for $P R$ were increased in PCL and ACL

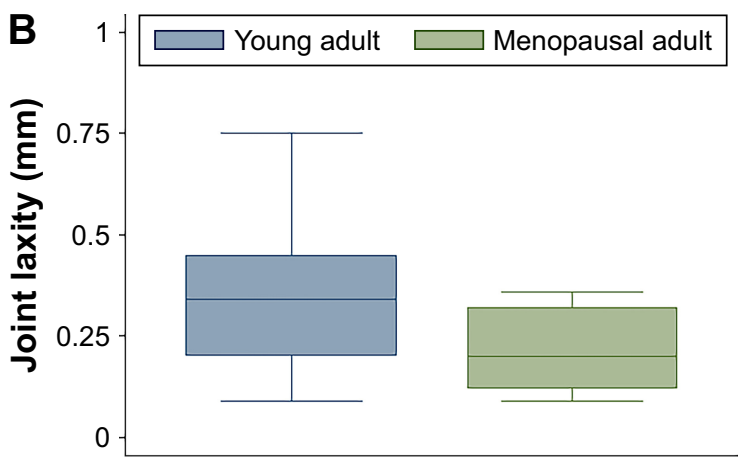


Table 3 Changes in mRNA levels with menopause

\begin{tabular}{|c|c|c|c|c|c|c|c|c|c|c|}
\hline Gene & FC AC & TP AC & MM & LM & Syn & $A C L$ & PCL & MCL & LCL & PT \\
\hline Collagen I & & & $\uparrow$ & & $\uparrow$ & & $\uparrow$ & $\uparrow$ & & $\uparrow$ \\
\hline Collagen III & & & $\uparrow$ & $\uparrow$ & & & $\uparrow$ & & & $\uparrow$ \\
\hline Collagen V & & $\downarrow$ & $\uparrow$ & & & & $\uparrow$ & & & \\
\hline Biglycan & & & & & $\downarrow$ & & & & & $\uparrow$ \\
\hline Decorin & & $\uparrow$ & & & & & & & & \\
\hline Lubricin & $\uparrow$ & & & & $\uparrow$ & $\uparrow$ & $\uparrow$ & $\uparrow$ & & \\
\hline HAS2 & & $\downarrow$ & & & $\downarrow$ & & & & & \\
\hline MMPI & & & & & $\uparrow$ & & $\uparrow$ & & $\downarrow$ & $\uparrow$ \\
\hline MMP3 & & & & & & & $\uparrow$ & & & \\
\hline MMPI3 & & & & & & & $\uparrow$ & & & \\
\hline TIMPI & & & & $\downarrow$ & $\uparrow$ & $\uparrow$ & & & & \\
\hline TIMP2 & & & & $\downarrow$ & $\downarrow$ & & & & & $\downarrow$ \\
\hline TIMP3 & & & & & & & $\uparrow$ & & & \\
\hline$E R$ & & & & & & & $\uparrow$ & & $\downarrow$ & \\
\hline$P R$ & & & & & & $\uparrow$ & $\uparrow$ & & & \\
\hline
\end{tabular}

Notes: Increase $(\uparrow)$ and decrease $(\downarrow)$ comparing tissues from menopausal adult rabbits to young adult rabbits $(p \leq 0.059)$. Summarizes the data shown in Figures S3 and S4. Abbreviations: ACL, anterior cruciate ligament; ER, estrogen receptor; FC AC, femoral condyle articular cartilage; HAS, hyaluronan synthase; LCL, lateral collateral ligament; LM, lateral meniscus; MMP, matrix metalloproteinase; MCL, medial collateral ligament; MM, medial meniscus; PCL, posterior cruciate ligament; PR, progesterone receptor; PT, patellar tendon; Syn, synovium; TP AC, tibial plateau articular cartilage; TIMP, tissue inhibitor of metalloproteinase.

( $P \leq 0.02$, Table 3$)$. Only the LCL demonstrated increased water content with surgical menopause $(P=0.001$, Figure $1 \mathrm{~B})$. No differences in joint laxity were detected between intact knee joints from young adult rabbits and menopausal adult rabbits (Figure 2B).

\section{Discussion}

Aging and menopause affected the gene-expression patterns of knee-joint tissues differently, suggesting unique changes to the set point, which is the cellular activity for homeostasis of the joint tissues to maintain joint function considering the joint as an organ. Interestingly, aging and menopause did not affect knee-joint laxity, suggesting that joint function was maintained, despite the multitude of changes in gene expression. Taken together, these findings support the theory of the joint as an organ, where the tissues of the joint adapt to maintain joint function. The young adult knee and the aging adult knee may be considered to have different set points, and an injury to the aging adult knee may have different consequences than the same injury to the young adult knee. The set point to maintain joint function has shifted due to aging, which may help explain why the same injury to an older knee joint becomes osteoarthritic faster than the same injury to a younger knee joint, as observed clinically. ${ }^{9}$

In our preclinical model, the set point for the knee joint changed with aging, with alteration in 37 of the 150 gene-tissue combinations evaluated (Table 2). The set point for the knee joint changed with surgical menopause, with alteration in 39 of the 150 gene-tissue combinations evaluated (Table 3). Despite the similar number of changes with aging (37 of 150) and menopause (39 of 150), only eleven changes were the same in both aging and menopause, which suggests unique overall changes to the set point, confirming that these physiologic processes must be considered independently in experimental models.

With aging, increases in MMP mRNA expression in FC AC, TP AC, and MM suggest the potential for extracellular matrix degradation that may not be balanced by concurrent increases in TIMPs in these same tissues, assuming the mRNA changes are ultimately reflected by changes in active enzymes. Increases in collagen expression in FC AC and collagen and proteoglycan expression in MM may attempt to counteract this degradation, but paradoxically in TP AC, collagen and proteoglycan expression decreased. The findings in this preclinical model are consistent with findings from human tissues, where increases in MMP1, MMP3, and MMP13 proteins with aging were documented in human AC and human chondrocyte cultures. ${ }^{18,19}$ Pathologic changes with aging are similar in human cartilage and menisci. ${ }^{8}$ With aging in human meniscus from knees without OA, the meniscal surface was intact, but the meniscal substance had abnormal matrix organization and cellularity. ${ }^{20}$ Although not evaluated in human meniscus with aging, nonhuman primate meniscus-explant cultures exhibited increased MMP1 and MMP3 in older degenerated versus younger healthy menisci. ${ }^{21}$ With menopause, FC AC, TP AC, and MM exhibited no changes in MMPs and TIMPs, but LM exhibited decreases in TIMPs. In AC, collagen was decreased (TP AC), 
but proteoglycans were increased (FC AC and TP AC). The MMP-TIMP imbalance in the LM may not be counteracted by the increase in collagen with menopause. While studies of molecular changes specifically attributable to menopause in human knee AC and meniscus are not available, Lou et $\mathrm{al}^{22}$ found that healthy postmenopausal women had more severe cartilage degeneration at the knee joint when compared with premenopausal and perimenopausal women grouped together, and Davies-Tuck et $\mathrm{al}^{23}$ reported that asymptomatic postmenopausal women who had meniscal tears were older and had more lateral cartilage degeneration.

Syn exhibited decreased biglycan mRNA levels with aging and menopause. With menopause, Syn exhibited decreased HAS2 and increased lubricin (proteoglycan 4, PRG4) expression. High-molecular-weight hyaluronan is synthesized by HAS2. ${ }^{24}$ Hyaluronan and lubricin act as joint lubricants in synovial joints. The relationship between HAS2 and lubricin (PRG4) was investigated in the temporomandibular joint of PRG4-knockout mice, where Koyama et $\mathrm{a}^{25}$ detected increased $H A S 2$ expression in chondrocytes, disc cells, and synoviocytes of PRG4-deficient mice. While decreased PRG4 was associated with increased HAS2 in synoviocytes of PRG4-knockout mice, we found decreased $H A S 2$ was associated with increased PRG4 in rabbit Syn with menopause, suggesting some attempt for either HAS2 or PRG4 to maintain some lubrication. Also, HAS2 was decreased in TP AC and LM with aging, but with no concurrent increase in lubricin. In human knee Syn, Yoshida et $\mathrm{al}^{24}$ reported decreased $H A S 2$ expression in OA patients (aged 29-59 years) compared with healthy donors (aged 45-83 years), and the authors commented that it was unclear whether the HAS difference was associated with age or arthritis pathology. Likewise, a portion of the OA patients and healthy donors were females, so the possible influence of menopause was also unclear.

With aging, the MCL and LCL exhibited decreased MMP expression, and the ACL, PCL, and MCL had increased TIMP expression. None of the ligaments exhibited changes in collagen expression with aging. Hasegawa et $\mathrm{al}^{26}$ found that human ACL had decreases in MMP1, MMP3, and MMP13 proteins with aging (TIMPs were not evaluated), where ACL from adults $>60$ years of age were classified as either aging ACL or degenerated ACL with moderate to severe ACL and cartilage degeneration. Taking the clinical and preclinical data together, these findings suggest that the MMP-TIMP imbalance in ligaments with aging may be unique to aging ligaments that are not yet overtly degenerating. With menopause, the LCL exhibited decreased MMP expression, and the ACL and PCL had increased TIMP expression. The PCL had changes in ten of 15 genes evaluated with menopause, including increases in the three MMPs and three collagens evaluated. With menopause, the ACL, PCL, and MCL had increased lubricin, which plays a role in the interfascicular lubrication of collagen in ligament. ${ }^{27}$ Increased lubricin may permit easier stretching of ligaments, which we confirmed in this same model of menopausal MCL and aging MCL with decreased modulus and increased failure strain. ${ }^{15,16}$ With menopause, the ACL and PCL exhibited increased $P R$. Decreased PR has been implicated in increased ligament stretch of the uterosacral ligament in pelvic-organ prolapse $\mathrm{e}^{28}$ and the pubic symphysis ligament in pregnancy. ${ }^{29}$ Increases in ligament stretch have been associated with increased lubricin and decreased PR. With menopause, the ACL and PCL had both increased lubricin and increased $P R$, which may have a net-zero effect on ligament stretch, consistent with the lack of change in joint laxity.

Only the PT exhibited decreased TIMP expression with aging (TIMP2). With menopause, the PT exhibited decreased TIMP 2 and increased $M M P 1$, collagen, and proteoglycan expression. In human degenerated PT, Parkinson et $\mathrm{al}^{30}$ found an increase in TIMP1 and no change in TIMP2 or MMP1. Given the differences in MMPs between aging ACL and degenerated ACL, ${ }^{26}$ it is possible that aging PT and degenerated PT have differences in MMPs and TIMPs. Morton et $\mathrm{al}^{31}$ did not find a difference in patellar tendinopathy status of premenopausal women compared with perimenopausal and postmenopausal women. Perhaps the changes in menopausal PT, like aging PT, may not be the same as changes in degenerated PT.

Despite a multitude of molecular changes in a variety of tissues, there was no resulting change in joint laxity in this preclinical model. A possible explanation is that the measurement of joint laxity used in this study may not have been sufficiently sensitive or was performed too early in the process of aging or menopause to detect any mechanical changes that could result from these molecular changes. However, an alternative explanation is that the changes in the joint are coordinated and synergistic, and together help to maintain homeostasis in the laxity of the joint and correspondingly joint function. Our observation of minimal water-content changes and no gross knee damage/degeneration further support the view that joint laxity was a suitable measure of joint function. Limited information is available on changes in joint laxity with aging and menopause in healthy human knees. When Sharma et $\mathrm{al}^{32}$ pooled the data from their control groups ("young" [aged 20-40 years] and "older" [aged 54-85 years]), they found that anterior-posterior laxity was not different, but varus-valgus laxity was greater for women than 
men, and that anterior-posterior laxity was not correlated with age, but varus-valgus laxity was weakly correlated $(r=0.29)$ with age. In a later study, Rudolph et $\mathrm{al}^{33}$ found no age-related change in varus-valgus laxity comparing data from two of their control groups ("middle-aged" [aged 40-57 years] and "old" [aged 60-80 years]). For our purposes, it is unfortunate that they did not measure radiographic varusvalgus laxity in their other control group ("young" [aged 18-25 years]). Taken together, these data suggest that joint laxity may only be weakly (or not at all) associated with aging, which supports the theory that the knee joint acts as an organ system to maintain joint function, even with molecular changes. It is unclear how any possible sex-related difference in joint laxity could be interpreted regarding menopauserelated differences specifically.

Because our objective was to investigate changes in the set point, which is the cellular activity for homeostasis of the joint tissues to maintain joint function considering the joint as an organ, we evaluated changes in gene expression only, but future examination of proteins and active enzymes also would be instructive further to support the joint as an organ theory. As detailed herein, many of the individual gene-expression changes in this preclinical model were similar to gene and protein changes reported in the clinical literature, and while carefully comparing this preclinical model to humans, the advantage of the preclinical model is the opportunity to evaluate the collective pattern of gene-expression changes in joint tissues under different conditions within a single model. Previous studies used the rabbit preclinical model as an aging model, starting with 2.6-year-old rabbits considered aged. ${ }^{34,35}$ In the rabbit preclinical model, decreased serumestrogen levels were found 2-14 weeks following ovariectomy surgery to induce surgical menopause. ${ }^{36-39}$ Although one explanation for our joint-laxity findings was that our measurement may not have been sufficiently sensitive, we have previously shown its sensitivity to joint instability, eg, PCL deficiency, ${ }^{17,40}$ which further supports the alternative explanation for our joint-laxity findings, ie, that changes in the joint tissues help to maintain homeostasis in joint laxity and correspondingly joint function.

The current study was successful in supporting the theory of the joint as an organ. Future studies could further support this theory by examining additional molecular markers and joint tissues, as well as investigating a larger-animal model and a temporal component. First, we investigated matrix molecules, proteinases and their inhibitors, and hormone receptors. Future work could include other molecular markers like inflammatory mediators that have been implicated in aging $^{41}$ and menopause. ${ }^{42}$ Including these mediators ensures a comparative baseline for aging or menopause with degenerative states, including $\mathrm{OA}$, which also has an important inflammatory component. ${ }^{41}$ Second, we investigated various joint tissues: AC, menisci, Syn, ligament and tendon. Future studies could examine bone and skeletal muscle because of the clinical conditions of osteoporosis and sarcopenia. Third, we used the rabbit model, and future studies might consider using a larger-animal model eg, the porcine model, which has been used to investigate aging ${ }^{43}$ and menopause. ${ }^{44,45}$ In fact, these studies in the porcine model investigated skeletalmuscle changes with aging ${ }^{43}$ and bone and cartilage changes with menopause, ${ }^{44,45}$ where calcium and vitamin D were evaluated due to hormonal and dietary influences. Fortunately, we have previously established a joint-laxity measurement in the porcine model and understand the advantages and disadvantages of comparing this model to humans. ${ }^{46}$ Last, we examined one time point of aging and one time point of menopause, and future studies should include a temporal component. The changes to the set point in the current study did not manifest in changes to joint laxity, indicating that adaptation of joint tissues was sufficient to maintain joint function. It would be interesting to determine whether longer exposure to the process of aging or menopause would result in a situation where the set point has changed so much that the adaptation of joint tissues is not sufficient and joint function is affected detrimentally.

\section{Conclusion}

Aging and menopause affected the gene-expression patterns of knee-joint tissues differently, suggesting unique changes to the set point of the knee. Interestingly, aging and menopause did not affect knee-joint laxity, suggesting that joint function was maintained, despite the changes in gene expression. Taken together, these findings support the theory of the joint as an organ where the tissues of the joint adapt to maintain joint function. The preclinical model had many findings that were similar to those published in the clinical literature, with the added benefit of direct comparison of joint tissues under different conditions within a single model. Future studies should further elucidate the normal processes of aging and menopause within the joint. It is important to remember that these processes are not synonymous, and must be considered when interpreting molecular data in pathologic knees.

\section{Acknowledgments}

The authors gratefully acknowledge the assistance of Dr Douglas W Morck, University Veterinarian. The authors also gratefully acknowledge the support of the Natural Sciences and Engineering Research Council of Canada 
(GMT: 261630/04560) and the Canadian Institutes of Health Research Institute of Musculoskeletal Health and Arthritis (GMT: 94006).

\section{Disclosure}

The authors report no conflicts of interest in this work.

\section{References}

1. Lo IK, Thornton G, Miniaci A, Frank CB, Rattner JB, Bray RC. Structure and function of diarthrodial joints. In: McGinty JB, editor. Operative Arthroscopy. 3rd ed. Philadelphia: Lippincott Williams and Wilkins; 2003:41-126.

2. Frank CB, Shrive NG, Boorman RS, Lo IK, Hart DA. New perspectives on bioengineering of joint tissues: joint adaptation creates a moving target for engineering replacement tissues. Ann Biomed Eng. 2004; 32(3):458-465.

3. Gao HL, Lin SQ, Wei Y, Chen Y, Wu ZL. The effect of age and menopausal status on musculoskeletal symptoms in Chinese women aged 35-64 years. Climacteric. 2013;16(6):639-645.

4. Central Intelligence Agency. Median age. 2017. Available from: https:// www.cia.gov/library/publications/the-world-factbook/fields/2177.html. Accessed September 1, 2017.

5. Felson DT, Lawrence RC, Dieppe PA, et al. Osteoarthritis: new insights - part 1: the disease and its risk factors. Ann Intern Med. 2000;133(8):635-646.

6. Srikanth VK, Fryer JL, Zhai G, Winzenberg TM, Hosmer D, Jones G. A meta-analysis of sex differences prevalence, incidence and severity of osteoarthritis. Osteoarthritis Cartilage. 2005;13(9):769-781.

7. Muraki S, Akune T, Oka H, et al. Incidence and risk factors for radiographic knee osteoarthritis and knee pain in Japanese men and women: a longitudinal population-based cohort study. Arthritis Rheum. 2012;64(5):1447-1456.

8. Loeser RH, Goldring SR, Scanzello CR, Goldring MB. Osteoarthritis: a disease of the joint as an organ. Arthritis Rheum. 2012;64(6): 1697-1707.

9. Roos H, Adalberth T, Dahlberg L, Lohmander LS. Osteoarthritis of the knee after injury to the anterior cruciate ligament or meniscus: the influence of time and age. Osteoarthritis Cartilage. 1995;3(4):261-267.

10. Ushiyama T, Ueyama H, Inoue K, Ohkubo I, Hukuda S. Expression of genes for estrogen receptors $\alpha$ and $\beta$ in human articular chondrocytes. Osteoarthritis Cartilage. 1999;7(6):560-566.

11. Ishizuka M, Hatori M, Suzuki T, et al. Sex steroid receptors in rheumatoid arthritis. Clin Sci (Lond). 2004;106(3):293-300.

12. Dietrich W, Haitel A, Holzer G, Huber JC, Kolbus A, Tschugguel W. Estrogen receptor- $\beta$ is the predominant estrogen receptor subtype in normal human synovia. J Soc Gynecol Investig. 2006;13(7):512-517.

13. Liu SH, al-Shaikh R, Panossian V, et al. Primary immunolocalization of estrogen and progesterone target cells in the human anterior cruciate ligament. J Orthop Res. 1996;14(4):526-533.

14. Sciore P, Frank CB, Hart DA. Identification of sex hormone receptors in human and rabbit ligaments of the knee by reverse transcriptionpolymerase chain reaction: evidence that receptors are present in tissue from both male and female subjects. J Orthop Res. 1998;16(5):604-610.

15. Thornton GM, Lemmex DB, Ono Y, et al. Aging affects mechanical properties and lubricin/PRG4 gene expression in normal ligaments. J Biomech. 2015;48(12):3306-3311.

16. Lemmex DB, Ono Y, Reno CR, Hart DA, Lo IK, Thornton GM. Increased lubricin/proteoglycan 4 gene expression and decreased modulus in medial collateral ligaments following ovariohysterectomy in the adult rabbit: evidence consistent with aging. J Biomech. 2016; 49(3):382-387.

17. Thornton GM, Shao X, Kuchison ME, Marchuk LL, Shrive NG, Frank CB. Healing ligament mechanical properties are improved by repair with interpositional allografts but not by concomitant treatment with hyaluronic acid. J Orthop Res. 2009;27(3):400-407.
18. Wu W, Billinghurst RC, Pidoux I, et al. Sites of collagenase cleavage and denaturation of type II collagen in aging and osteoarthritic articular cartilage and their relationship to the distribution of matrix metalloproteinase 1 and matrix metalloproteinase 13. Arthritis Rheum. 2002; 46(8):2087-2094.

19. Dozin B, Malpeli M, Camardella L, Cancedda R, Pietrangelo A. Response of young, aged and osteoarthritic human articular chondrocytes to inflammatory cytokines: molecular and cellular aspects. Matrix Biol. 2002;21(5):449-459.

20. Pauli C, Grogan SP, Patil S, et al. Macroscopic and histopathologic analysis of human knee menisci in aging and osteoarthritis. Osteoarthritis Cartilage. 2011;19(9):1132-1141.

21. Stone AV, Vanderman KS, Willey JS, et al. Osteoarthritic changes in vervet monkey knees correlate with meniscus degradation and increased matrix metalloproteinase and cytokine secretion. Osteoarthritis Cartilage. 2015;23(10):1780-1789.

22. Lou C, Xiang G, Weng Q, et al. Menopause is associated with articular cartilage degeneration: a clinical study of knee joint in 860 women. Menopause. 2016;23(11):1239-1246.

23. Davies-Tuck ML, Martel-Pelletier J, Wluka AE, et al. Meniscal tear and increased tibial plateau bone area in healthy post-menopausal women. Osteoarthritis Cartilage. 2008;16(2):268-271.

24. Yoshida M, Sai S, Marumo K, et al. Expression analysis of three isoforms of hyaluronan synthase and hyaluronidase in the synovium of knees in osteoarthritis and rheumatoid arthritis by quantitative real-time reverse transcriptase polymerase chain reaction. Arthritis Res Ther. 2004; 6(6):R514-R520.

25. Koyama E, Saunders C, Salhab I, et al. Lubricin is required for the structural integrity and post-natal maintenance of TMJ. J Dent Res. 2014;93(7):663-670.

26. Hasegawa A, Nakahara H, Kinoshita M, Asahara H, Koziol J, Lotz MK. Cellular and extracellular matrix changes in anterior cruciate ligaments during human knee aging and osteoarthritis. Arthritis Res Ther. 2013;15(1):R29.

27. Funakoshi T, Schmid T, Hsu H, Spector M. Lubricin distribution in the goat infraspinatus tendon: a basis for interfascicular lubrication. J Bone Joint Surg Am. 2008;90(4):803-814.

28. Bai SW, Chung DJ, Yoon JM, Shin JS, Kim SK, Park KH. Roles of estrogen receptor, progesterone receptor, p53 and p21 in pathogenesis of pelvic organ prolapse. Int Urogynecol J Pelvic Floor Dysfunct. 2005; 16(6):492-496.

29. Rodríguez HA, Ramos JG, Ortega HH, Muñoz-de-Toro M, Luque EH. Regional changes in the spatio-temporal pattern of progesterone receptor (PR) expression in the guinea-pig genital tract as parturition approaches. J Steroid Biochem Mol Biol. 2008;111(3-5):247-254.

30. Parkinson J, Samiric T, Ilic MZ, Cook J, Feller JA, Handley CJ. Change in proteoglycan metabolism is a characteristic of human patellar tendinopathy. Arthritis Rheum. 2010;62(10):3028-3035.

31. Morton S, Williams S, Valle X, Diaz-Cueli D, Malliaras P, Morrissey D. Patellar tendinopathy and potential risk factors: an international database of cases and controls. Clin J Sport Med. 2017;27(5):468-474.

32. Sharma L, Lou C, Felson DT, et al. Laxity in healthy and osteoarthritic knees. Arthritis Rheum. 1999;42(5):861-870.

33. Rudolph KS, Schmitt LC, Lewek MD. Age-related changes in strength, joint laxity, and walking patterns: are they related to knee osteoarthritis? Phys Ther. 2007;87(11):1422-1432.

34. Duan W, Wei L, Zhang J, et al. Alteration of viscoelastic properties is associated with a change in cytoskeleton components of ageing chondrocytes from rabbit knee articular cartilage. Mol Cell Biomech. 2011; 8(4):253-274.

35. Guzman-Morales J, Lafantaisie-Favreau CH, Chen G, Hoemann CD. Subchondral chitosan/blood implant-guided bone plate resorption and woven bone repair is coupled to hyaline cartilage regeneration from microdrill holes in aged rabbit knees. Osteoarthritis Cartilage. 2014; 22(2):323-333.

36. Aikawa K, Sugino T, Matsumoto S, Chichester P, Whitbeck C, Levin RM. The effect of ovariectomy and estradiol on rabbit bladder smooth muscle contraction and morphology. J Urol. 2003;170(2 Pt 1):634-637. 
37. Komatsuda T, Sugita T, Sano H, et al. Does estrogen alter the mechanical properties of the anterior cruciate ligament? An experimental study in rabbits. Acta Orthop. 2006;77(6):973-980.

38. Yang F, Hu K, Wang X, et al. Combination of raloxifene, aspirin and estrogen as novel paradigm of hormone replacement therapy in rabbit model of menopause. Acta Pharmacol Sin. 2011;32(8):1031-1037.

39. Abramov Y, Hirsch E, Ilievski V, Goldberg RP, Sand PK. Transforming growth factor $\beta 1$ gene expression during vaginal wound healing in a rabbit menopause model. BJOG. 2013;120(2):251-256.

40. Thornton GM, Bailey SJ, Chamberland NM. Spontaneous posterior cruciate ligament deficiency decreases the strength of healing rabbit medial collateral ligaments. Poster presented at: 55th Annual Meeting of the Orthopaedic Research Society; February 22-25, 2009; Las Vegas, NV.

41. Greene MA, Loeser RF. Aging-related inflammation in osteoarthritis. Osteoarthritis Cartilage. 2015;23(11):1966-1971.
42. Pfeilschifter J, Köditz R, Pfohl M, Schatz H. Changes in proinflammatory cytokine activity after menopause. Endocr Rev. 2002;23(1):90-119.

43. Jin L, Jiang Z, Xia Y, et al. Genome-wide DNA methylation changes in skeletal muscle between young and middle-aged pigs. BMC Genomics. 2014; $15: 653$.

44. Claassen H, Cellarius C, Scholz-Ahrens KE, et al. Extracellular matrix changes in knee joint cartilage following bone-active drug treatment. Cell Tissue Res. 2006;324(2):279-289.

45. Pufe T, Claassen H, Scholz-Ahrens KE, et al. Influence of estradiol on vascular endothelial growth factor expression in bone: a study in Göttingen miniature pigs and human osteoblasts. Calcif Tissue Int. 2007;80(3):184-191.

46. Germscheid NM, Thornton GM, Hart DA, Hildebrand KA. A biomechanical assessment to evaluate breed differences in normal porcine medial collateral ligaments. J Biomech. 2011;44(4):725-731. 


\section{Supplementary materials}
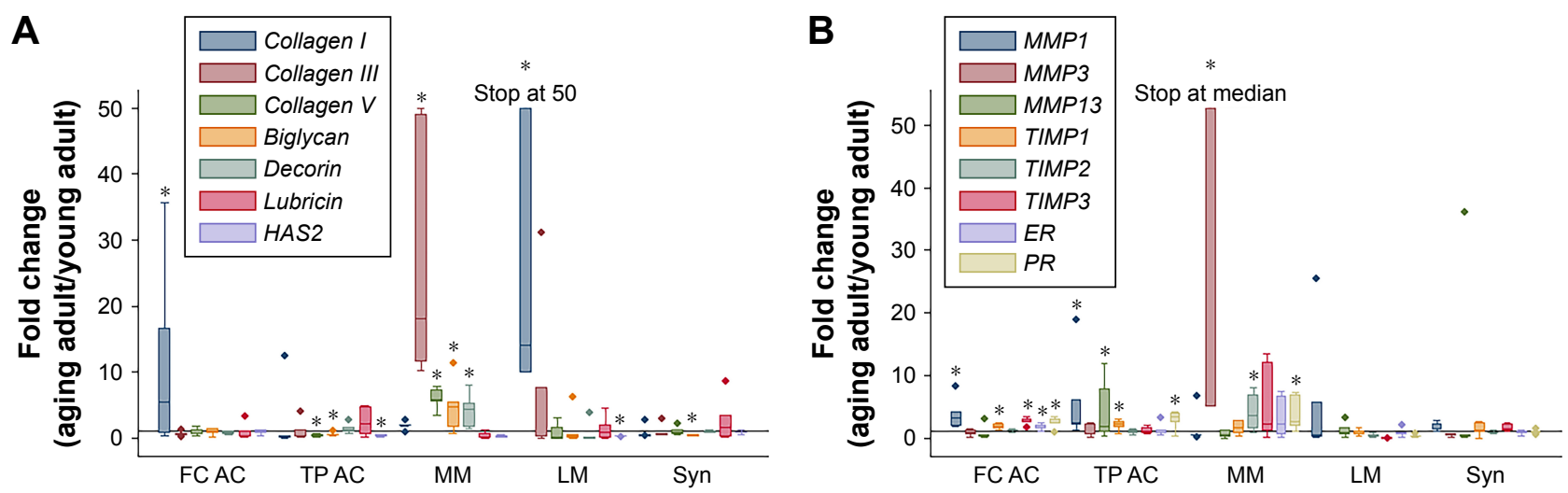

Figure SI Fold change in mRNA levels with aging (aging adult/young adult) for cartilage, meniscus, and synovium: (A) collagens, proteoglycans, and HAS2; (B) MMPs, TIMPs, and hormone receptors

Notes: $* P \leq 0.059$. The horizontal reference line represents a fold change of $\mathrm{I}$. The diamond symbol on the box plot indicates an outlier.

Abbreviations: ER, estrogen receptor; FC AC, femoral condyle articular cartilage; HAS, hyaluronan synthase; LM, lateral meniscus; MM, medial meniscus; MMP, matrix metalloproteinase; PR, progesterone receptor; Syn, synovium; TIMP, tissue inhibitor of metalloproteinase; TP AC, tibial plateau articular cartilage.

A

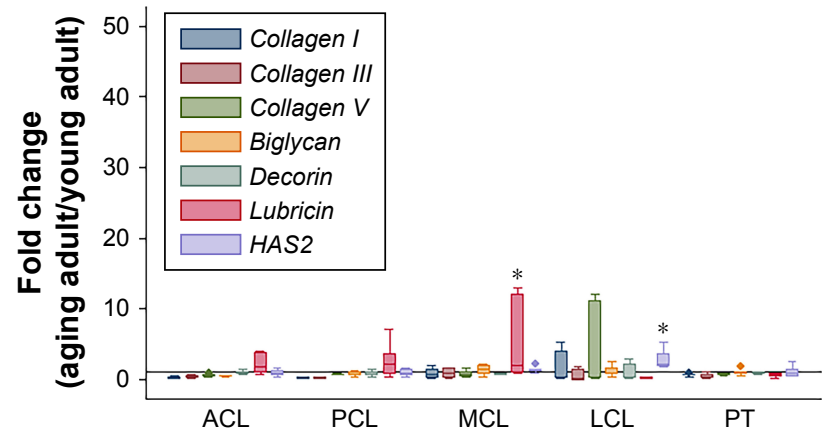

B

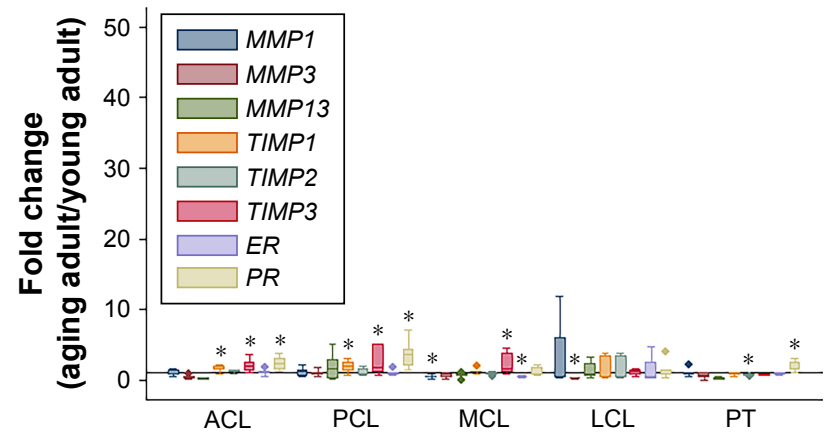

Figure S2 Fold change in mRNA levels with aging (aging adult/young adult) for ligament and tendon: (A) collagens, proteoglycans, and HAS2; (B) MMPs, TIMPs, and hormone receptors.

Notes: $* P \leq 0.059$. The horizontal reference line represents a fold change of $\mathrm{I}$. The diamond symbol on the box plot indicates an outlier.

Abbreviations: ACL, anterior cruciate ligament; ER, estrogen receptor; HAS, hyaluronan synthase; LCL, lateral collateral ligament; MCL, medial collateral ligament; MMP, matrix metalloproteinase; PCL, posterior cruciate ligament; PR, progesterone receptor; PT, patellar tendon; TIMP, tissue inhibitor of metalloproteinase.
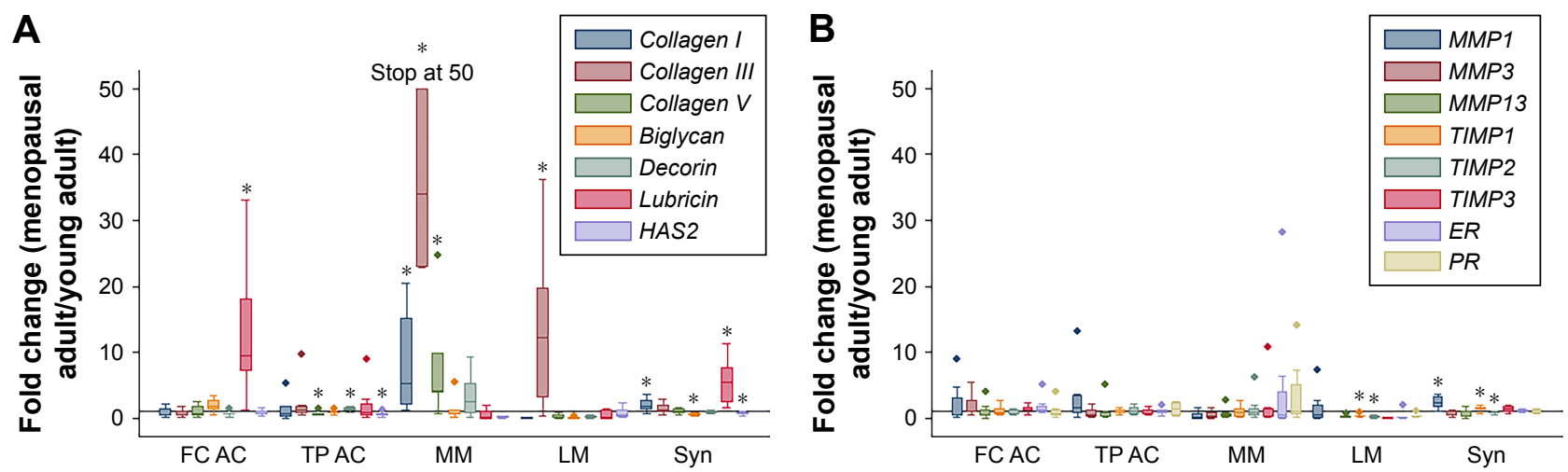

Figure S3 Fold change in mRNA levels with menopause (menopausal adult/young adult) for cartilage, meniscus, and synovium: (A) collagens, proteoglycans, and HAS2; (B) MMPs, TIMPs, and hormone receptors.

Notes: $* P \leq 0.059$. The horizontal reference line represents a fold change of $I$. The diamond symbol on the box plot indicates an outlier.

Abbreviations: ER, estrogen receptor; FC AC, femoral condyle articular cartilage; HAS, hyaluronan synthase; LM, lateral meniscus; MM, medial meniscus; MMP, matrix metalloproteinase; PR, progesterone receptor; Syn, synovium; TIMP, tissue inhibitor of metalloproteinase; TP AC, tibial plateau articular cartilage. 
A

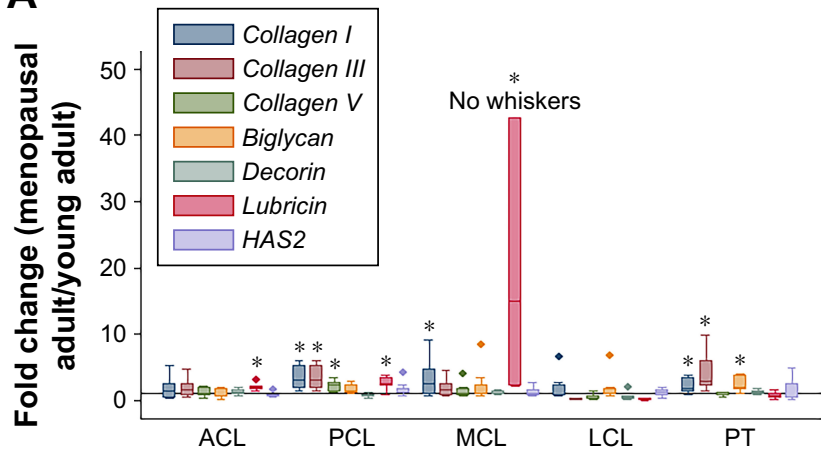

B

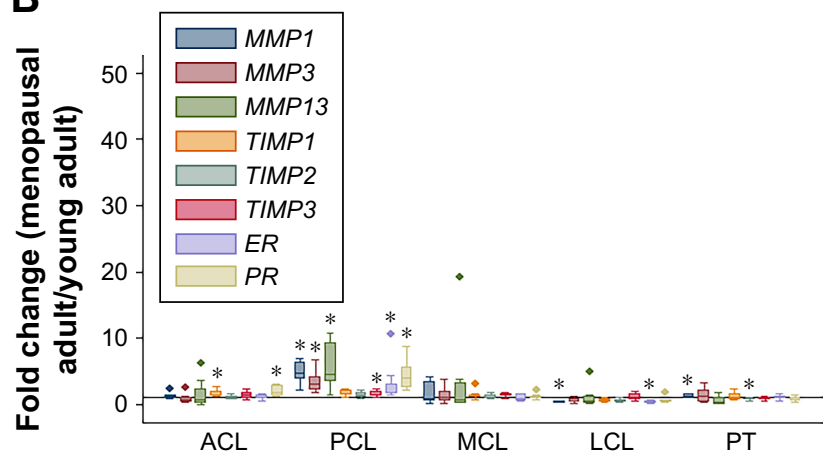

Figure S4 Fold change in mRNA levels with menopause (menopausal adult/young adult) for ligament and tendon: (A) collagens, proteoglycans, and HAS2; (B) MMPs, TIMPs, and hormone receptors.

Notes: $* P \leq 0.059$. The horizontal reference line represents a fold change of $I$. The diamond symbol on the box plot indicates an outlier.

Abbreviations: ACL, anterior cruciate ligament; ER, estrogen receptor; HAS, hyaluronan synthase; LCL, lateral collateral ligament; MCL, medial collateral ligament; MMP, matrix metalloproteinase; PCL, posterior cruciate ligament; PR, progesterone receptor; PT, patellar tendon; TIMP, tissue inhibitor of metalloproteinase.

\section{Publish your work in this journal}

Clinical Interventions in Aging is an international, peer-reviewed journal focusing on evidence-based reports on the value or lack thereof of treatments intended to prevent or delay the onset of maladaptive correlates of aging in human beings. This journal is indexed on PubMed Central, MedLine,
CAS, Scopus and the Elsevier Bibliographic databases. The manuscript management system is completely online and includes a very quick and fair peer-review system, which is all easy to use. Visit http://www.dovepress. $\mathrm{com} /$ testimonials.php to read real quotes from published authors. 